\title{
Inspired by Emotions, Guided by Knowledge: Which Emotional Cues Dominate Knowledge Management Research?
}

\author{
Olivia Hornung \\ University of Hagen, Germany \\ olivia.hornung@fernuni-hagen.de
}

\author{
Nora Fteimi \\ University of Passau, Germany \\ nora.fteimi@uni-passau.de
}

\author{
Stefan Smolnik \\ University of Hagen, Germany \\ stefan.smolnik@fernuni-hagen.de
}

\begin{abstract}
Knowledge, being context-specific and bound to individuals, is strongly related to human emotions such as joy or fear. Although emotions play an important role to articulate knowledge in text, KM research only offers insight on emotions from specific angles, neglecting a holistic view. Applying a sentiment analysis, this study closes the aforementioned gap by investigating the occurrence of emotions in KM publications. Based on general sentiment dictionaries, we (1) develop a dictionary aligned with KM, and (2) apply it to KM publications to determine the presence of positive and negative emotions and categorize them according to an emotion scale. Our results reveal that a variety of emotions is expressed in KM studies, both positive and negative, proving its relevance for this domain. We find that there is high term diversity, but also the need for consolidation of terms as well as emotion categories in $K M$.
\end{abstract}

\section{Introduction}

Classic Greek philosopher Plato once said: "Human behavior flows from three main sources: desire, emotion, and knowledge." Research on knowledge management (KM) has proven to be of strong organizational relevance, since successful KM leads to significant improvements of scientific, economic and social aspects [6]. Knowledge is often merely viewed as another organizational resource, but due to its context-specificity and boundedness to human beings [37], it cannot be separated from human emotions. Thus, the role of emotions, which both help to express and understand knowledge [9], requires encompassing attention from KM researchers as well as within the information systems (IS) domain in general.
IS researchers have recently started to pay attention to the presence and role of emotions $[4,18$, 19].With regard to KM processes, the role of emotional intelligence $[10,39,52]$ or emotional obstacles $[31,38]$ have been investigated. While these studies constitute an invaluable stake on the way to acknowledge emotions and the role emotional concepts play for KM, these studies show how heterogeneous KM research on emotions is. Thus, there is need for consolidation of research on single emotions and emotional concepts and in which nexus they displayed in KM research - with a taxonomy of emotions in KM research as the final goal. To arrive at a comprehensive taxonomy of emotions in KM, we will have to obtain an overview, which emotional cues - ultimately serving as an indicator for emotions - are used in KM research and in which intensity and frequency they occur. Gaining a first understanding of emotions by finding and analyzing emotional cues can be achieved through sentiment analyses - which have often been used to detect emotional words in the context of social media or marketing [34, 57]. Sentiment analyses, which constitute an application field of text mining, are helpful for our endeavor as "analyzing the sentiment of a unit of text can encompass investigating both the opinion and the emotion behind that unit" [56]. In this study, we take the first step towards ultimately uncovering and understanding the role of emotions in KM research by addressing the following research question:

Which and how many emotional cues prevail and dominate in existing KM research?

We used the sentiment dictionaries by $\mathrm{Hu}$ and Liu [22] and modified them to better suit KM research endeavors. Herewith, we will not only be able to detect which emotions prevail in existing KM research, but also to contribute to future research efforts in this domain by (1) developing a KM-specific sentiment dictionary and (2) applying this dictionary to English KM journals' publications as the majority of publications are written in English [42]. Subsequently, 
the results of our sentiment analysis are manually categorized and grouped according to emotion scales to classify and structure the results according to appropriate emotional categories, thus providing a preparatory step towards the taxonomy planned for the future.

Next, we provide an overview on different streams of literature concerning emotions theories in KM. Then, we describe our research approach and present the results. The paper concludes with a discussion and implications for research and practice.

\section{Research background}

\subsection{Emotion theories}

Emotions serve as the primary motivational system for human beings $[28,36]$, leading to an emotional component in virtually any action and interaction of human beings - including communication through information systems [43]. Emotions as research object have heavily been investigated by researchers of the psychology discipline, not leading to one universal but many different definitions and conceptualizations [7, 14]. However, ambiguity on this matter has not kept researchers from various disciplines to take on further investigations regarding emotions and their role in and impact on corresponding research domains.

Definitions range from the rather general point of view that emotions direct cognitive activities $[8,32]$ to more specific ones stating that emotions are the complex derived reactions to a stimulus [41]. In our study, emotions are seen as a sequence which chronologically unfolds: a human being is exposed to a stimulus, perceives a state of "feeling," and, consequently, displays externally visible behaviors or emotional outputs [13]. While the concept of emotion is not fully congruent with other concepts such as mood or feeling [45], these are still strongly related concepts and often used interchangeably in extant research [5]. Therefore, we will initially include what we classify as emotions, feelings, moods, and sentiments in our study to grasp the full extent of emotion-related words in KM research and then assign each of these words to an appropriate category.

In order to substantially embed our research in existing emotion theory, using a profound model can help with emotion classification as "the distinction between positive and negative emotions is fundamental in emotion models" [3]. While there are several well-established models in research, some have a wide spectrum of not clearly positive or negative emotions, and include many emotions that are not relevant in a KM-context, such as Plutchik's wheel of emotion [40] or Richins' consumptionrelated emotions [44]. Other models, such as the computer emotion scale by Kay and Loverock [24], have a strong focus on negative emotions, which is unfavorable for exploratory studies. Thus, we decided to apply a well-established model by Izard [23], called differential emotion scale (DES), encompassing the following ten emotions: interest, joy, surprise, sadness, anger, disgust, contempt, fear, shame, and guilt.

\subsection{Emotions in knowledge management research}

KM comprises all conscious and organized efforts to develop, preserve and utilize knowledge to add value, achieve goals and improve an organization's situation [21]. Apart from having high practical significance, KM is a well-established discipline with many journals and conference tracks dedicated to investigating and advancing academic KM research [46]. From 1993, when the KM discipline emerged, until 2012, there were $12,925 \mathrm{KM}$-related publications [42] - a number further increasing to this date.

KM researchers within the IS domain have used a vast array of research methods and approaches [11] to examine KM theories, processes and technologies [15]. KM research on emotions has been insightful, but either specific to one single emotion, such as trust $[47,48]$ or pride [55], or focusing on related concepts such as emotional intelligence [10, 16, 53]. More studies investigated positive emotions as contributor to successful $\mathrm{KM}[1,33,49,51]$ than negative emotions as hindrance to successful KM use and outcomes [31, 39], which is why we decided to conduct a comprehensive investigation and classification of both positive and negative emotions.

With increasing popularity to analyze opinions and emotions in domains such as politics, finance, or marketing research [34], sentiment dictionaries listing positive and negative terms were developed. They constitute one possible approach to deal with sentiment expressions and make the exploration of vast amounts of data convenient and exploiting. A sentiment is an opinion or idea tinted by an emotion, making it possible to reveal an emotion through the sentiment analysis of a text unit [56].

Yet, text mining analysis in $\mathrm{KM}$ has not necessarily focused on emotions, but on uncovering different KM topics [42]. For instance, Ur-Rahman and Harding investigated the mechanics behind text analysis for organizational KM [54]. Furthermore, to better understand terms frequently used in KM research, (reference removed) developed a dictionary 
specific to KM using text mining approaches. Albeit being popular to analyze social media data and research [26, 57], a domain connected to $\mathrm{KM}$, sentiment dictionaries have not been used with regard to $\mathrm{KM}$ research to the best of our knowledge.

\section{Research process and methods}

The paper relies on a sentiment analysis applying a dictionary-based approach (also known as a bag-ofwords' model), which uses a mapping algorithm to compare the text with the content of the dictionary [30]. We proceeded in three steps (c.f. Figure 1).

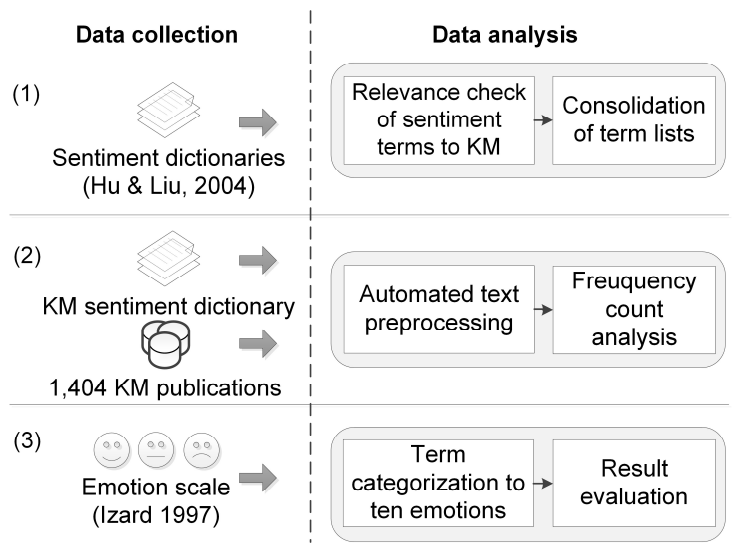

Figure 1. Multi-step research approach

In step 1, we manually developed the sentiment dictionaries. Step 2 included the application of these dictionaries to KM data using text analytical approaches. We then, in step 3, evaluated the analyses' results by looking at the positive and negative terms' occurrence frequencies and consolidated them to emotional scale categories to arrive at an overview how many and which emotional cues dominate KM research.

\section{Step 1: sentiment dictionary development}

First, we selected dictionaries to be adapted for the purpose of this study. We decided to use the dictionaries of $\mathrm{Hu}$ and Liu [22], which cover two extensive lists of positively and negatively connoted terms and, thus, ensure suitability for a wide range of application domains. The original lists of terms include 2,007 positively and 4,783 negatively connoted terms separated according to two different lists.

Since dictionaries should always be used in an awareness of the respective context [25, 34], we needed to verify and reduce both term lists for their adaptability in the KM context. Therefore, the lists were coded by two of the authors to identify the topical relevance of each term to KM. We removed the terms which we did not consider to be relevant to KM from the lists with an intercoder-reliability value of 0.7 for the positive term list and 0.68 for the negative term list. Both values indicate a strong reliability regarding the coding agreement between both coders [27]. Eventually, we consolidated the coding results by removing all terms that were eliminated by both coders. The adaptation and reduction of the original dictionaries led to two separate lists containing 1,860 positively and 4,092 negatively connoted terms customized to the purposes of KM. We reduced the negative term list by almost $20 \%$ percent - hence, twice as much as the positive term list - because the negative term list contained more colloquial slang words than the positive list. Due to the academic and formal nature of our research object (abstracts of research publications), slang or colloquial wording seemed unlikely to occur and, thus, was not applicable to our sample. We used these customized lists as an input for the subsequent sentiment analysis in step 2 .

\section{Step 2: text mining analysis}

In order to investigate the role of emotions in KM, we applied the dictionaries developed in step 1 to 1,404 KM publications. We decided to first analyze two selected KM journals. Based on the latest KM journal ranking [46], the $(A+)$ ranked Journal of Knowledge Management (JKM) and the $(A)$ ranked International Journal of Knowledge Management (IJKM) were selected for analysis. Both outlets are specialized KM journals and premier publication targets for $\mathrm{KM}$ researchers and practitioners with vigorous publication activities.

We obtained our data from the database Scopus and included all available publications' titles and abstracts (until March 2018). Editorials were excluded from the analysis, as they do not provide this metadata. The overall dataset comprised 1,155 articles published in the $J K M$ and 249 articles published in the IJKM. The differences in volume size are due to the annual publication intensity of both journals and the year of their inaugural issue.

Next, we conducted the analysis using the statistical computing software $\mathrm{R}$, which is a free software environment that provides different packages and functions to handle large data volumes and, among various applications, is well suited to perform text mining [2]. The corpus consists of the titles and abstracts of all analyzed publications. A title and its corresponding abstract represent one dataset within the corpus.

To ensure that the data is processed correctly, some pre-processing text mining steps are necessary [12]. 
This includes, for example, the harmonization of all letters to lower case as well as the removal of all nontextual tokens (e.g. numbers and punctuation marks). We applied these transformations to the corpus. In addition, we eliminated some specific stop-word sequences and expressions, which, due to the journals' structural requirements, appeared in each dataset. For instance, abstracts in the JKM typically include the term sequence "design - methodology - approach" or "research limitations - implications". Since individual terms from these sequences also appear in the sentiment dictionaries (see step 1), we removed these sequences to avoid bias in the analysis' results.

Subsequently, we applied the sentiment dictionaries to the data in order to find the occurrence of each positive and negative term from the dictionaries in the corpus. The software R operates as follows: it compares each term in the dictionary to the corpus and once a match is identified, the term's frequency count is automatically updated. Afterwards, we consolidated and evaluated the results in step 3.

\section{Step 3: interpretation of results}

We started the last step of our study by consolidating terms that have similar meanings, but occur in different forms, to a single term. Furthermore, we summed up their frequency counts (e.g. trust, trustworthiness, and trustworthy were consolidated to trust).

To further summarize the results and categorize them into a taxonomy of KM emotions, we started to categorize each term according an emotion scale. As stated in our background section, we used the DES by Izard, who proposes to distinguish between the following three basic positive emotion categories: interest, joy, and surprise, and the following seven basic negative emotions: anger, contempt, disgust, fear, guilt, sadness, and shame [23]. According to Izard, all other positive or negative emotions are gradations of these ten [23]. We began to assign each term of the frequency count lists to one of these basic categories. Like in step 1 , the assignment was performed independently by two of the authors. We introduced an additional category called (N/A) in order to account for terms that cannot be meaningfully assigned to one of the ten basic emotions. During the assignment, we referred to the definitions of these basic emotional terms from The Oxford English Dictionary [50], which provides appropriate definitions for each. Additionally, we searched for synonyms of identified terms on a thesaurus (http://www.thesaurus.com). All terms for which we could not reach an agreement regarding their categorization (we reached a significant intercoderreliability value of 0.5 for the classification of positive terms and a weak value of 0.3 for the negative term classification) were additionally categorized by a third expert. Furthermore, we conducted team discussions to identify and select a suitable category for ambiguous assignments. This was particularly necessary for the negative term list, as the coding results often led to different categories.

\section{Emotion analysis of knowledge management publications}

The analysis led to a frequency count list of all positive and negative terms, which were identified through matching the dictionaries' terms to our KM corpus. These show how many and which emotional cues dominate KM research.

\subsection{Text mining analysis of positive terms}

The results revealed that in effect only 507 (27\%) positively connoted terms from the positive dictionary list were used in the analyzed KM articles. After consolidating the list with regard to different spellings (c.f. section 3.3), the number of non-redundant terms was reduced to a summary of 330 terms and their consolidated count frequencies of totally 9,557 counts.

The ten most frequent positive terms are innovation (800 counts) followed by effective ( 573 counts), support (528 counts), success (475 counts), improve (385 counts), important (298 counts), best (295 counts), well (283 counts), positive (219 counts), beneficial (203 counts). Of the 330 terms, 22 terms (7\%) were mentioned more than 100 times with a relative frequency count of 63\%. 97 terms (29\%) occurred between 10 and 99 times (relative frequency count: 30\%), and the remaining 211 terms (64\%) were mentioned less than ten times. Of the last group, 72 terms occurred only once in the corpus. In summary, positively connoted terms are used in $97 \%$ of the analyzed data sets, indicating a high degree of coverage. Only 41 datasets do not contain any of the positive terms.

\subsection{Text mining analysis of negative terms}

With 446 terms mentioned, the analysis indicates a slightly lower number of negative terms than the analysis of positive terms. The consolidation of this initial list with regard to different spellings leads to a reduced one of totally 305 non-redundant terms and their consolidated frequencies $(3,661$ count frequencies).

The top ten most frequently mentioned negative terms are limit (1,288 counts) followed by critic ( 236 
counts), problem (183 counts), risk (140 counts), complex (117 counts), lack (113 counts), difficult (74 counts), fail (74 counts), lose (68 counts) and negative (62 counts). The previous elimination of stop words and specific sequences, which are part of the mandatory structure of some abstracts (c.f. section 3), ensures that these terms were only counted if they occurred as a single term in the corpus. Of the 305 terms, only six $(2 \%)$ achieved a frequency count higher than 100 (relative frequency count: 57\%). In contrast, 261 negatively connoted terms occurred between 10 and 99 times with a relative frequency count of $26 \%$. Of the remaining 261 terms $(86 \%)$ that were used less than 10 times, 125 ones occurred once.

Compared to the coverage of positive connoted terms over the corpus, negative connoted terms appeared only in $88 \%$ of the datasets, which means that 162 datasets did not exhibit negative terms at all.

\subsection{Term classification according to emotion scales}

As a result of the subsequent classification process, each of the 330 positive terms and 305 negative terms were manually categorized into one of the ten emotional categories joy, interest, surprise, anger, contempt, disgust, fear, guilt, sadness, and shame. In case an unambiguous categorization was not possible, we introduced the category $N / A$.

Due to the similar meanings of the categories contempt and disgust, we decided to merge them into a combined category called contempt/disgust. We took this decision during the classification process, as it turned out that many of the terms that would have been categorized into one of these two categories often could not be solely assigned to one single emotion. In addition, the category disgust included finally only nine terms. Therefore, we opted for a merging for better comprehensibility. The same applies to the two categories shame and guilt (the category guilt included finally only four terms), which were also considered a common category.

Table 1 provides a meta-summary of all categories according to positive and negative emotion scales.

Table 1. Meta-summary of emotional scales

\begin{tabular}{|l|c|c|}
\hline \multicolumn{3}{|l|}{ Positive emotion scales } \\
\hline Category & $\begin{array}{l}\text { Term } \\
\text { share }\end{array}$ & $\begin{array}{l}\text { Frequency } \\
\text { count }\end{array}$ \\
\hline Interest & $45 \%$ & $64 \%$ \\
\hline Joy & $40 \%$ & $29 \%$ \\
\hline Surprise & $8 \%$ & $3 \%$ \\
\hline
\end{tabular}

\begin{tabular}{|l|c|c|}
\hline N/A & $7 \%$ & $4 \%$ \\
\hline \multicolumn{2}{|l|}{ Negative emotion scales } \\
\hline Contempt / Disgust & $38 \%$ & $60 \%$ \\
\hline Anger & $18 \%$ & $7 \%$ \\
\hline Fear & $17 \%$ & $15 \%$ \\
\hline Sadness & $12 \%$ & $5 \%$ \\
\hline Shame/Guilt & $6 \%$ & $11 \%$ \\
\hline$N / A$ & $9 \%$ & $2 \%$ \\
\hline
\end{tabular}

For each category presented in Table 1, the percentage of all its terms to the total number of all positive (or negative) terms is specified (column Term share). The column Frequency count indicates the relative occurrence frequencies for all terms of a respective category compared to the sum of all positive (or negative) occurrence frequencies.

4.3.1. Positive emotional categories in knowledge management publications. The most comprehensive positive category is interest with a term share of $45 \%$ and a relative frequency count summary of $64 \%$ (relative to all frequency counts of positive words). The upper word cloud in Figure 2 provides an overview of the top positive terms assigned to this category. A term's size indicates its frequency count.

We observe that the interest category covers four of the five most frequent positive terms. However, the majority of terms in this category $(91 \%)$ were mentioned less than 100 times. Terms in this category express a helpful or important feeling, which can draw interest or attention on something. For instance, an innovative KM solution or improvement can be helpful for an organization's growth and, thus, causes interest to implement it. Terms like support, effective, beneficial, or talent all have the same positive connotation, expressing a helpful feeling.

The second category covers all positively connoted terms that are related to joy with a term share of $40 \%$ and a relative frequency count summary of $29 \%$ (relative to all frequency counts). The lower word cloud in Figure 2 visualizes the top terms assigned to this category. With 475 counts, the term success was mentioned the most, followed by best (295 counts), well (283 counts), positive (219 counts), and lead (178 counts).

Similar to the first category, the majority of terms $(95 \%)$ were mentioned less than 100 times. A closer look at the categories reveals that many terms can express a positive aspect and, thus, suggest joy or happiness. This observation can be confirmed, particularly by terms such as success, advantage, or intelligence, which are commonly targeted in $\mathrm{KM}$ publications. A successful implementation of a KM 
system or valuable feedback within KM initiatives can evoke joy or satisfaction. Also, a harmonious culture can also lead to satisfied employees.

The remaining two categories surprise and $N / A$ each cover only 24 terms ( $7 \%$ term share and a relative frequency count in summary below $5 \%$ ) and are therefore not considered relevant for this study (and, hence, also not depicted in Figure 2).

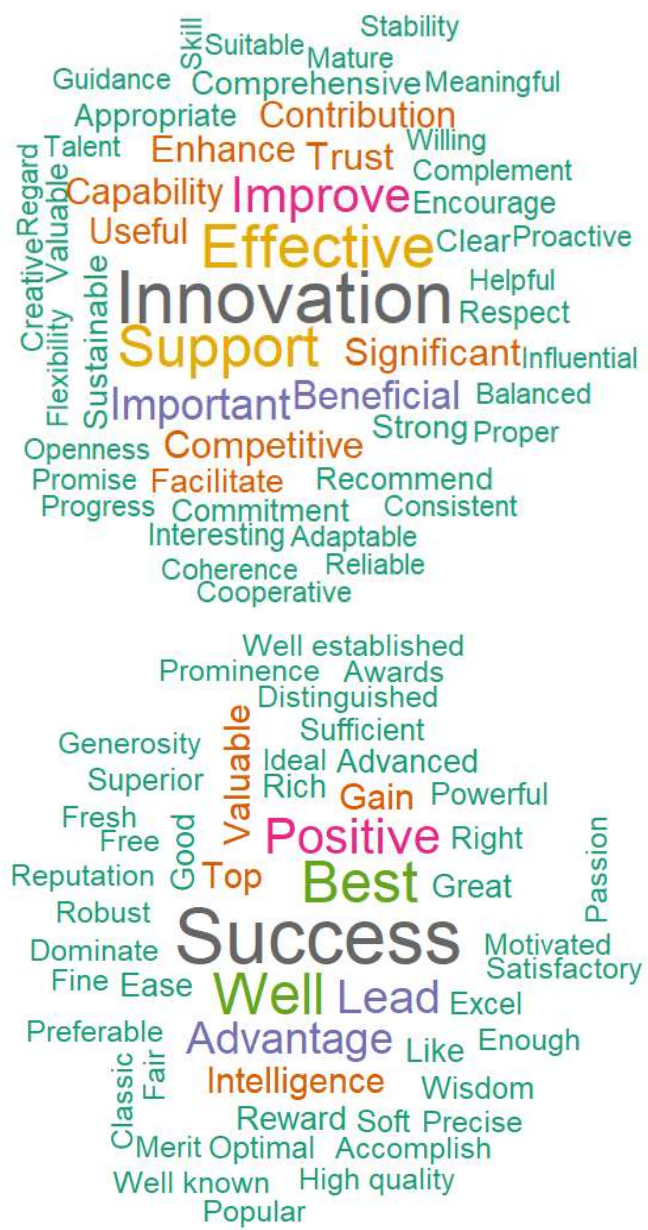

Figure 2. Top positive terms for the emotions "interest" (top) \& “joy" (bottom)

4.3.2. Negative emotional categories in knowledge management publications. With a total term share of $38 \%$, the category contempt/disgust is the most comprehensive one, followed by the categories anger, fear, sadness, shame/guilt and N/A.

Similar to the positive categories, we focus our descriptions and illustrations (c.f. Figures 3 and 4) to those categories that have reached a term share higher than $10 \%$. Therefore, shame/guilt and N/A are not visualized here. In each of the four negative word clouds, a term's size indicates its respective frequency count. The first word cloud is the sole one that contains more than 100 terms and is limited for visualization purposes to the top terms in this category.

Both emotions contempt and disgust express the feeling that a certain situation or thing is despised or disregarded and is therefore worthless or beneath consideration [50]. Exemplary terms, which are associated with this category, are problem, reject, mistrust, unknown, inappropriate or useless. In the context of KM such a despised situation could arise if a problem or deviating or even unknown result occurs, for example, during the implementation of a KM tool, resulting in the system losing value. Even insufficient or inappropriate results or a slow system or user performance may lead to a lower appreciation of results or even their rejection.

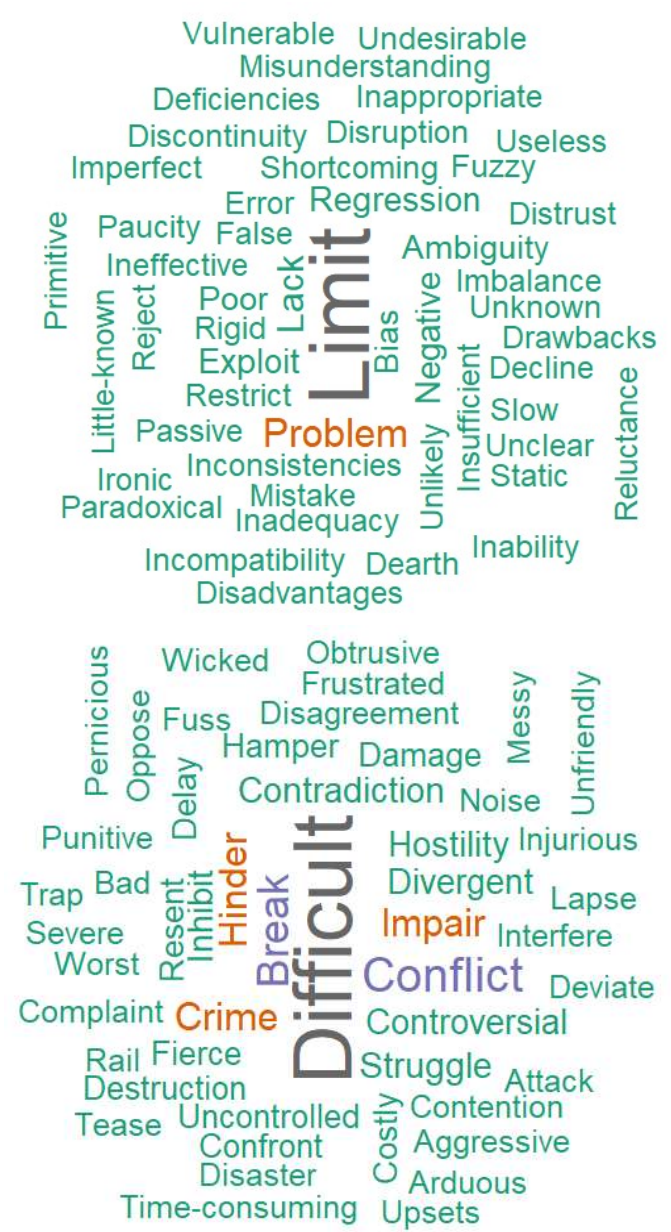

Figure 3. Top negative terms for the emotions "contempt/disgust" (top) \& "anger" (bottom)

The category labelled anger indicates a strong feeling of annoyance or displeasure [50]. A difficult 
situation, some hindering circumstances, a conflict in a team of users or a costly implementation can all evoke a feeling of anger. Time-consuming tasks, delays in meeting schedules or system attacks can also cause trouble and be therefore associated with anger. However, we have observed that terms in this category have not been used as frequently as in the previous category. The term list is led by "difficult" with 74 counts, followed by "conflict (25 counts)" and "break (19 counts)".

With terms like risk, complex, challenging, dilemma, or danger, the third category encompassing the emotion fear describes those emotions than can evoke an unwelcome or even anxious feeling - thus leading someone to be afraid or scared [50]. The top term in this list is risk (140 counts), followed by complex (117 counts) and concern (47 counts) on the second and third ranks.

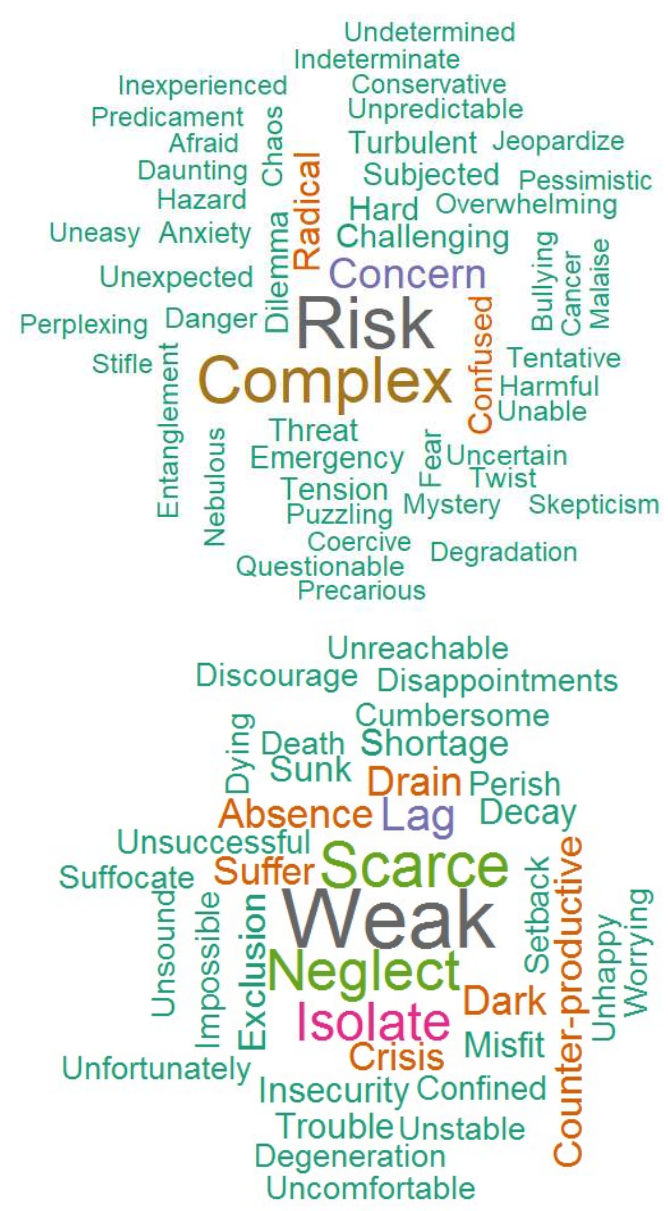

Figure 4. Top negative terms for the emotions "fear" (top) and "sadness" (bottom)
The last word cloud visualizes all 38 terms $(12 \%$ term share) that we have associated with the feeling of sadness including terms like weak (34 counts), scarce (20 counts), isolate (15 counts), or lag (10 counts). For instance, sadness may be caused by feelings of isolation or failure to realize projects or plans regarding $\mathrm{KM}$.

\section{Discussion}

Our results reveal that emotions emotional cues exist in KM research. More specifically, particular terms (e.g. success, innovation and trust for the positive terms or problem, risk and difficult for the negative terms), which are highly associated with topics dealing with the successful or failed implementation of KM initiatives and processes, occur quite often in $\mathrm{KM}$ publications.

In summary, we observed that KM researchers attempt to primarily use words that indicate an undesirable situation, which when related to $\mathrm{KM}$, is associated with the deployment of technologies, the implementation of relating KM strategies, or the establishment of an organization-wide KM culture. Furthermore, such feelings can occur when an unexpected outcome leads to disappointment. However, words describing anger, fear, and sadness are depicted less vigorously in scientific texts, possibly be due to the more intense expressiveness of such emotions.

Additionally, some positive words (e.g. beneficial, helpful, and useful) as well as many negative words (e.g. problem, error, and mistake) are identified as synonyms, which allow drawing conclusions on term diversity - but also the need for term consolidation in KM. This ambivalence becomes visible in our findings, which suggest a higher frequency of positively connoted terms in KM publications (97\%) than negatively connoted terms $(88 \%)$, yet the size of the negatively connoted sentiment dictionary $(4,092$ words) encompasses more than twice as many expressions as the positively connoted sentiment dictionary.

Furthermore, our attempt for consolidation is subject to the chosen emotion scale, which offers more negative than positive basic emotions. Many other emotion scales either provide a strong focus on negative sentiments [24] or encompass many interpersonal emotions $[40,44]$ that are unlikely to occur in scientific KM publications. Hence, the DES [23] is offering a good basis for emotion research in KM. Nonetheless, especially the positive basic emotions mostly represented in only two categories, interest and joy, suggest that KM ultimately needs its 
own taxonomy of emotions with more diverse positive categories. Furthermore, the KM-specific emotion taxonomy should encompass fewer negative categories than the DES suggests, as we merged contempt with disgust as well as shame with guilt.

In our results, we see that many emotions, specifically those which are stronger or not typically researched in KM are under-represented and may provide examples for white spots and possible areas for future research. Especially for categories that we did not depict in the word clouds due to lack of counts, such as the positively connoted surprise, further investigations could be conducted, since the negatively connoted counterpart fear has received such vast attention with two of the top negative sentiment words, complex and risk, in this category.

In further studies, we intend to broaden our analysis particularly by using a more comprehensive dataset to develop a deeper and generalizable taxonomy of emotions in KM research. Thereby, we aim to help identifying current research gaps and generate implications for future actions and research in this domain. To achieve this aim, we will deepen the text mining analysis in the next step by applying machine learning techniques to our corpus (e.g. topic modeling [35]). This can provide further interesting and more reliable results than a manual classification technique by building emotional topic categories that group the related sentiment terms based on the content of documents. Furthermore, we will compare the current methodological approach with machine learning approaches by performing the analysis using appropriate classification algorithms on training data sets (e.g. using support vector machines [20]).

\section{Conclusion}

As part of our overall research project to investigate the role of emotions in KM research and arrive at an overall taxonomy, this study aims to present the results of developing a KM-specific sentiment dictionary and its application to $\mathrm{KM}$ publications using text mining methods. The first steps towards the intended emotions-in-KM taxonomy were taken by identifying positive and negative emotional cues in KM research and manually categorizing them according to an emotion scale. Herewith, we were able to show which emotions so far dominate KM research.

Limitations of our study concern the missing context during the text mining analysis and the manual effort taken during the categorization process. Some terms in the positive categories can also express a negative emotion or feeling (e.g. enough, classic, simpler). The context, in which a term is used, is thus important and may affect the interpretation and meaning of such terms. A statement like enough liquid funds may express joy but in another context like enough problems a feeling of anger or contempt. The same applies for terms from the negative categories, which, depending on the situation, may be sometimes interpreted as a positive feeling, too (lower costs $\rightarrow$ joy category vs. lower motivation $\rightarrow$ anger category). For our current study, we refer to the already predefined categorization based on the applied sentiment dictionaries, according to which each occurring sentiment term has either a positive or a negative connotation. A possible future solution to this challenge is to carry out the text analysis following a two-step approach and to first make an initial evaluation and classification of particular emotionally laden text excerpts. Using this information as a basis, the factual emotion analysis could be done in the second step. Furthermore, our approach, especially the consolidation and coding of terms, is time-consuming and relies on the judgement and efforts of all involved analysts.

With this attempt to unveil emotions in $\mathrm{KM}$ research, we have contributed to several research streams in IS. Albeit the nature of knowledge being strongly tied to emotions and sentiments, we are the first to use sentiment analysis in a KM context to the best of our knowledge. By modifying the sentiment dictionaries to suit the KM context and then classification them into the DES, our study is also the first attempt to apply the DES to KM research, although the comparison with the analyses' results of the machine learning approach is still pending. We have also contributed to emotion research in KM by giving a comprehensive overview of emotions and emotional cues in KM research. Hereby, we have shown the need for consolidation of emotions in KM and the need for a taxonomy of KM emotions to explain relations and connections in the KM context.

Regarding our theoretical contribution in the IS context, our research contributes to the analysis as described by Gregor's [17] theory types in IS research. Developing a taxonomy and applying it to research objects generally serves the purpose to systematically describe these research objects [29] according to specific common dimensions or attributes. Here, our envisioned emotions-in-KM taxonomy is terminologically descriptive and allows for classification of sentiment expressions, which are the research objects in this study. This study represents the first steps towards a comprehensive framework which then will give causal explanations, proceeding a step further in said IS theory type taxonomy [17]. 


\section{References}

[1] Aarrestad, M., M.T. Brøndbo, and A. Carlsen, "When Stakes are High and Guards are Low: High-quality Connections in Knowledge Creation", Knowledge and Process Management, 22(2), 2015, pp. 88-98.

[2] http://cran.r-project.org/doc/manuals/R-intro.pdf, accessed 4-25-2018.

[3] Aviezer, H., Y. Trope, and A. Todorov, "Body cues, not facial expressions, discriminate between intense positive and negative emotions", Science (New York, N.Y.), 338(6111), 2012, pp. 1225-1229.

[4] Beaudry, A. and A. Pinsonneault, "The Other Side of Acceptance: Studying the Direct and Indirect Effects of Emotions on Information Technology Use", Management Information Systems Quarterly, 34(4), 2010, pp. 689-710.

[5] Beedie, C., P. Terry, and A. Lane, "Distinctions between emotion and mood", Cognition \& Emotion, 19(6), 2005, pp. 847-878.

[6] Cao, X., D.R. Vogel, X. Guo, H. Liu, and J. Gu, "Understanding the Influence of Social Media in the Workplace: An Integration of Media Synchronicity and Social Capital Theories", in $201245^{\text {th }}$ Hawaii International Conference on System Science: (HICSS) ; USA, 4 - 7 Jan. 2012, R.H. Sprague, Editor, $201245^{\text {th }}$ Hawaii International Conference on System Sciences (HICSS), Maui, HI, USA, 4/1/2012 - 7/1/2012. 2012. IEEE: Piscataway, NJ.

[7] Chaplin, J.S. and T.S. Krawiec, "Systems and Theories of Psychology", University of Illinois Press, Champaign, IL, 1979.

[8] Clark, M.S. and S.T. Fiske, Affect and cognition: The seventeenth annual Carnegie symposium on cognition, Psychology Press, 1982.

[9] Davenport, T.H. and L. Prusak, Working knowledge: How organizations manage what they know, Harvard Business Press, 1998.

[10] Decker, B., R.E. Landaeta, and T.G. Kotnour, "Exploring the relationships between emotional intelligence and the use of knowledge transfer methods in the project environment", Knowledge Management Research \& Practice, 7(1), 2009, pp. 15-36.

[11] Dwivedi, Y.K., K. Venkitachalam, A.M. Sharif, W. AlKaraghouli, and V. Weerakkody, "Research Trends in Knowledge Management: Analyzing the Past and Predicting the Future", Information Systems Management, 28(1), 2011, pp. 43-56.

[12] Elder, J., G. Miner, and B. Nisbet, Practical text mining and statistical analysis for non-structured text data applications, $1^{\text {st }}$ edn., Academic Press, Amsterdam, 2012.

[13] Elfenbein, H.A., "Emotion in Organizations", The Academy of Management Annals, 1(1), 2007, pp. 315-386.
[14] English, H.B. and A.C. English, "A comprehensive dictionary of psychological and psychoanalytical terms: A guide to usage”, Longmans, Green, 1958.

[15] Fteimi, N. and F. Lehner, "Main Research Topics in Knowledge Management: A Content Analysis of ECKM Publications", Electronic Journal of Knowledge Management, 14(1), 2016, pp. 5-17.

[16] Geofroy, Z. de and M.M. Evans, "Are Emotionally Intelligent Employees Less Likely to Hide Their Knowledge?", Knowledge and Process Management, 24(2), 2017, pp. 81-95.

[17] Gregor, S., "The Nature of Theory in Information Systems", MIS Quarterly, 30(3), 2006, p. 611.

[18] Gregor, S., A.C.H. Lin, T. Gedeon, A. Riaz, and D. Zhu, "Neuroscience and a Nomological Network for the Understanding and Assessment of Emotions in Information Systems Research", Journal of Management Information Systems, 30(4), 2014, pp. 13-48.

[19] Guinea, A.O.d., R. Titah, and P.-M. Léger, "Explicit and Implicit Antecedents of Users' Behavioral Beliefs in Information Systems: A Neuropsychological Investigation", Journal of Management Information Systems, 30(4), 2014, pp. 179-210.

[20] Hearst, M.A., S.T. Dumais, E. Osuna, J. Platt, and B. Scholkopf, "Support vector machines", IEEE Intelligent Systems and their Applications, 13(4), 1998, pp. 18-28.

[21] Holsapple, C.W. and K.D. Joshi, “A formal knowledge management ontology: Conduct, activities, resources, and influences", Journal of the American Society for Information Science and Technology, 55(7), 2004, pp. 593-612.

[22] Hu, M. and B. Liu, "Mining and summarizing customer reviews", in Proceedings of the tenth ACM SIGKDD international conference on Knowledge discovery and data mining, Seattle, USA. 2004. ACM: New York.

[23] Izard, C.E., "Differential Emotions Theory", in Human Emotions. 1977. Springer US: Boston, MA.

[24] Kay, R.H. and S. Loverock, "Assessing emotions related to learning new software: The computer emotion scale", Computers in Human Behavior, 24(4), 2008, pp. $1605-1623$.

[25] Krippendorff, K., Content analysis: An introduction to its methodology, Sage, Los Angeles, London, New Delhi, Singapore, 2013.

[26] Kumar, A. and T.M. Sebastian, "Sentiment analysis on twitter", IJCSI International Journal of Computer Science Issues, 9(3), 2012.

[27] Landis, J.R. and G.G. Koch, "The Measurement of Observer Agreement for Categorical Data", Biometrics, 33(1), 1977, p. 159.

[28] Leeper, R.W., "A motivational theory of emotion to replace "emotion as disorganized response.", Psychological Review, 55(1), 1948, pp. 5-21. 
[29] Lehner, F., „Grundfragen und Positionierung der Wirtschaftsinformatik“, Wirtschaftsinformatik: Theoretische Grundlagen. München, 1995, pp. 1-72.

[30] Li, F., "The Information Content of Forward-Looking Statements in Corporate Filings-A Naïve Bayesian Machine Learning Approach", Journal of Accounting Research, 48(5), 2010, pp. 1049-1102.

[31] Lin, J., H.C. Chan, and K.K. Wei, "The effects of goal orientations on knowledge management system usage, knowledge sourcing and learning outcome", in ECIS. 2006.

[32] Mandler, G., Mind and emotion, Krieger Publishing Company, 1975.

[33] Marshall, J., "Expanding the realm of organizational reasoning", The Learning Organization, 7(5), 2000, pp. 244-251.

[34] Matthies, B., "Feature-based sentiment analysis of codified project knowledge: A dictionary approach", PACIS 2016 Proceedings, 2016.

[35] Mcauliffe, J.D. and D.M. Blei, "Supervised Topic Models", in Advances in Neural Information Processing Systems 20, J.C. Platt, D. Koller, Y. Singer, and S.T. Roweis, Editors. 2008. Curran Associates, Inc.

[36] Mowrer, O.H., Learning theory and behavior, John Wiley \& Sons Inc, Hoboken, NJ, US, 1960.

[37] Nonaka, I., "A dynamic theory of organizational knowledge creation", Organization science, 5(1), 1994, pp. 14-37.

[38] Pemberton, J., S. Mavin, and B. Stalker, "Scratching beneath the surface of communities of (mal)practice", The Learning Organization, 14(1), 2007, pp. 62-73.

[39] Peng, H., "Why and when do people hide knowledge?", Journal of Knowledge Management, 17(3), 2013, pp. 398415.

[40] Plutchik, R., A general psychoevolutionary theory of emotion, Academic Press, New York, 1980.

[41] Plutchik, R., "Emotions: A general psychoevolutionary theory", Approaches to emotion, 1984, pp. 197-219.

[42] Qiu, J. and H. Lv, "An overview of knowledge management research viewed through the web of science (1993-2012)", Aslib Journal of Information Management, 66(4), 2014, pp. 424-442.

[43] Rice, R.E. and G. Love, "Electronic Emotion: Socioemotional Content in a Computer-Mediated Communication Network", Communication Research, 14(1), 1987, pp. 85-108.

[44] Richins, M.L., "Measuring Emotions in the Consumption Experience", Journal of Consumer Research, 24(2), 1997, pp. 127-146.

[45] Rottenberg, J., "Mood and Emotion in Major Depression", Current Directions in Psychological Science, 14(3), 2005, pp. 167-170.
[46] Serenko, A. and N. Bontis, "Global ranking of knowledge management and intellectual capital academic journals: 2017 update", Journal of Knowledge Management, 21(3), 2017, pp. 675-692.

[47] Song, S. and J.T.C. Teng, "An Exploratory Examination of Knowledge Sharing Behaviors: Voluntary vs. Solicited", in Proceedings of the $41^{\text {st }}$ Annual Hawaii International Conference on System Sciences (HICSS) 2008, Waikoloa, HI, USA, 7/1/2008 - 10/1/2008. 2008. IEEE.

[48] Swift, P.E. and A. Hwang, "The impact of affective and cognitive trust on knowledge sharing and organizational learning", The Learning Organization, 20(1), 2013, pp. 20 37.

[49] Tenório, N., A. Ferrarezi Vidotti, M. Alaranta, and H.K. Fulk, "The Influence of Positive Emotions on Knowledge Sharing”, AMCIS 2017 Proceedings, 2017, pp. 1-5.

[50] The Oxford English Dictionary: On CD-ROM ; including additions series volumes $1-3,2^{\text {nd }}$ edn., Oxford Univ. Press, Oxford, 2007.

[51] Trenck, A.v.d., F. Emamjome, T. Neben, and A. Heinzl, "What's in it for Me? Conceptualizing the Perceived Value of Knowledge Sharing", in $48^{\text {th }}$ Hawaii International Conference on System Sciences (HICSS), 2015: 5 - 8 Jan. 2015, Kauai, Hawaii, T.X. Bui and R.H. Sprague, Editors, $201548^{\text {th }}$ Hawaii International Conference on System Sciences (HICSS), HI, USA, 5/1/2015 - 8/1/2015. 2015. IEEE: Piscataway, NJ

[52] Trong Tuan, L., "Leading to learning and competitive intelligence", The Learning Organization, 20(3), 2013, pp. 216-239.

[53] Tuan, L.T., "The chain effect from human resourcebased clinical governance through emotional intelligence and CSR to knowledge sharing", Knowledge Management Research \& Practice, 14(1), 2016, pp. 126-143.

[54] Ur-Rahman, N. and J.A. Harding, "Textual data mining for industrial knowledge management and text classification: A business oriented approach", Expert Systems with Applications, 39(5), 2012, pp. 4729-4739.

[55] van den Hooff, B., A.P. Schouten, and S. Simonovski, "What one feels and what one knows: The influence of emotions on attitudes and intentions towards knowledge sharing", Journal of Knowledge Management, 16(1), 2012, pp. $148-158$

[56] Yadollahi, A., A.G. Shahraki, and O.R. Zaiane, "Current State of Text Sentiment Analysis from Opinion to Emotion Mining", ACM Computing Surveys, 50(2), 2017, pp. 1-33.

[57] Yassine, M. and H. Hajj, "A Framework for Emotion Mining from Text in Online Social Networks", in IEEE International Conference on Data Mining workshops (ICDMW), 2010: Sydney, Australia, 13 [i.e. 14] Dec. 2010 ; proceedings, W. Fan, Editor, 2010 IEEE International Conference on Data Mining Workshops (ICDMW), Sydney, TBD, Australia. 2010. IEEE: Piscataway, NJ. 